\title{
PFLEGE FÜR PATIENTEN MIT DIABETISCHEN FUß
}

\section{ORIGINALER ARTIKEL}

CUNHA, Amanda Priscilla da ${ }^{1}$

CUNHA, Amanda Priscilla da. Pflege für Patienten mit diabetischen Fuß. Revista Científica Multidisciplinar Núcleo do Conhecimento. Jahr. 06, Hrsg. 12, Vol. 12, S. 111-126. Dezember 2021. ISSN: 2448-0959, Zugangslink: https://www.nucleodoconhecimento.com.br/gesundheit/diabetischen-fuss, DOI: 10.32749/nucleodoconhecimento.com.br/gesundheit/diabetischen-fuss

\section{ZUSAMMENFASSUNG}

Einleitung: Der diabetische Fuß, der auch durch einen facettenreichen pathophysiologischen Zustand bezeichnet wird, ist durch Läsionen gekennzeichnet, die ohne angemessene Behandlung und spezifische Pflege von den Füßen der Person mit Diabetes ausgehen. Es wird geschätzt, dass die Inzidenz von diabetischen Fußgeschwüren 6,3\% der Diabetiker erreicht und ihre Prävalenz sich $10 \%$ nähert, und Menschen mit niedrigem sozioökonomischen Status sind die größten Opfer. Angesichts dieser Evidenz basiert die Arbeit auf folgender Problemfrage: Welche Pflege entwickeln Pflegekräfte bei Patienten mit diabetischen Füßen? Allgemeines Ziel: Nachweis der Versorgung des diabetischen Fußes im Zusammenhang mit der Praxis von Krankenschwestern. Methodik: Es wurde ein integratives Review durchgeführt, bei dem in der Auf Pflege spezialisierte bibliographische Datenbank (BDENF), in der Lateinamerikanische und karibische Literatur in den Gesundheitswissenschaften (LILACS) und in recherchiert wurde der Scientific Electronic Library Online (SCIELO) und die zu sieben Artikeln führte.. Ergebnisse: Die Studien zeigten, wie die Pflege von Krankenschwestern mit diabetischer Fuß Gesundheitserziehung, Fort- und Weiterbildung,

\footnotetext{
${ }^{1}$ Master in Krankenhausmanagement und Gesundheitssystemen, Krankenschwester Full Degree in Krankenpflege. ORCID: 000-0001-5456-1848.
}

RC: 105166

Verfügbar in: https://www.nucleodoconhecimento.com.br/gesundheit/diabetischenfuss 
Bewertungstechniken zum diabetischen Fuß, die körperliche Untersuchungen und Selbstversorgung von Patienten mit diabetischen Füßen beinhalten. Es wird bekräftigt, dass diese Pflege vorrangig von Krankenschwestern durchgeführt werden sollte, da Krankenschwestern in ihren Haupt Zuordnungen eine spezifische und qualifizierte Versorgung des Patienten haben. Schlussfolgerung: Es wird daher der Schluss gezogen, dass die Studie mehrere Formen der Pflege durch Krankenschwestern identifiziert hat und dass alle diese Formen für die Kontinuität der Behandlung und die Vermeidung von Komplikationen wichtig sind, jedoch liegt es an der Krankenschwester, ein Fachmann zu sein, der die Qualität ihrer Pflege auf der Grundlage theoretischer Konzepte schätzt, die zu häufigen Schulungen führen.

Schlüsselwörter: Patientenversorgung, Diabetes mellitus, Diabetischer Fuß.

\section{EINLEITUNG}

Diabetes mellitus (DM) ist eine im endokrinen System entwickelte Krankheit und/oder eine chronische Stoffwechselstörung, die ein ernstes weltweites Gesundheitsproblem darstellt und als Hauptmerkmal eine Hyperglykämie aufweist, die beim Fasten einen Blutzuckerspiegel von mehr als $126 \mathrm{mg} / \mathrm{dl}$ oder im postprandialen Stadium >200 $\mathrm{mg} / \mathrm{dl}$ erreicht und die möglicherweise mit Insulinresistenz in Verbindung gebracht wurde und die mit Insulinresistenz in Verbindung gebracht wurde. unzureichende Insulinsekretion oder übermäßige Glucagonsekretion (ASCHNER et al., 2016).

Daher verursacht Hyperglykämie eine vollständige oder teilweise Unfähigkeit der Insulinproduktion, nach der brasilianischen Diabetes-Gesellschaft (SBD), der American Diabetes Association (ADA) und der Weltgesundheitsorganisation (WHO) ist DM in vier Gruppen unterteilt: Diabetes mellitus Typ 1, Diabetes mellitus Typ 2, Schwangerschaftsdiabetes mellitus und andere spezifische Arten von Diabetes (OMS, 1999; SBD, 2017; ADA 2017).

SBD beschreibt Risikofaktoren für DM, die wie folgt zitiert werden: Diagnose von Prädiabetes - verminderte Toleranz gegenüber Glukose oder veränderte 
Nüchternglukose; Bluthochdruck; Hypercholesterinämie oder Veränderungen der Triglyceridwerte im Blut; Übergewicht und Fettleibigkeit, besonders wenn Fett um die Taille konzentriert ist; Familiengeschichte; Baby mit einem Gewicht von mehr als vier Kilo oder Schwangerschaftsdiabetes; polyzystisches Ovarialsyndrom; Diagnose einiger psychiatrischer Störungen wie Schizophrenie, Depression, bipolare Störung und Verwendung von Psychopharmaka; andere (SBD, 2018).

Da es sich um eine Krankheit handelt, von der etwa $3 \%$ der Weltbevölkerung betroffen sind, und mit einer ungefähren Prävalenz von 6,2 \% der Bevölkerung in Brasilien, deren Prävalenz aufgrund der Bevölkerungsalterung zunimmt (MUZY et al., 2021), hat DM eine Belastung verursacht aus den öffentlichen Kassen, zusätzlich zu den hohen Kosten für die Durchführung der Behandlung seiner Komplikationen wie: diabetischer Fuß, Herz-Kreislauf-Erkrankungen, Amputation und andere. Es ist erwähnenswert, dass die pharmakologische Behandlung Fortschritte bei der Bekämpfung von DM gemacht hat, jedoch muss die Gesundheitserziehung als eine Form der Prävention an die allgemeine Bevölkerung einzeln oder in Gruppen weitergegeben werden, wobei das Problem der Krankheit und ihre Gesundheitsschäden betont werden (SILVA, 2018).

In Bezug auf die Komplikationen von DM wird der diabetische Fuß auch durch seinen facettenreichen pathophysiologischen Zustand bezeichnet, der durch Läsionen gekennzeichnet ist, die in 90\% der Fälle aus den Füßen der Person mit Diabetes aus Neuropathie entstehen, in denen periphere Gefäßerkrankungen und Deformitäten vorherrschen (CUBAS et al., 2017).

De Sousa Mendonça; Moraes; Moura (2017) beschreibt, dass die bestehenden Läsionen im diabetischen Fuß von der Kombination von zwei oder mehr Risikofaktoren herrühren, die mit intrinsischen Traumata wie extrinsisch zusammenhängen können, die mit einer peripheren Neuropathie verbunden sind, die einen Verlust der Empfindlichkeit verursacht und das Risiko von Schnitten erleichtert; Periphere Gefäßerkrankung, die sich aus einem verminderten Blutfluss in den unteren Gliedmaßen und einer biomechanischen Veränderung ergibt, die auftritt, 
wenn die Bewegungseinschränkungen der Gelenke des Fußes und des Knöchels eingeschränkt sind.

Es wird geschätzt, dass die Inzidenz von diabetischen Fußgeschwüren 6,3\% der Diabetiker erreicht und ihre Prävalenz sich 10\% nähert, und Menschen mit niedrigem sozioökonomischen Status sind die größten Opfer. Die Amputation des diabetischen Fußes ist ein weiteres Problem und dass weltweit alle drei Minuten einem Menschen sein Glied aufgrund von DM amputiert wird (ARRUDA et al., 2021).

Das gute Management von Faktoren, die an der Ätiologie der bestehenden Läsion im diabetischen Fuß beteiligt sind, wird wirksam, wenn es von der Krankenschwester durchgeführt wird, so dass die Krankenschwester qualifiziert und qualifiziert sein muss, die Pflegeberatung durchzuführen, um dermatologische, muskuloskelettale, vaskuläre und neurologische Veränderungen im diabetischen Fuß zu übersehen (FÉLIX et al., 2021).

Daher wird die Pflege von diabetischen Füßen in der öffentlichen Gesundheit sehr wichtig und die Krankenschwester, als Fachmann, der auf seiner Prämisse die Pflege des Patienten hat, sollte aufmerksam und qualifiziert in Bezug auf die neuen Behandlungsformen sein, um Amputationen zu reduzieren. Angesichts dieses Szenarios alarmierender Raten ist es wichtig zu beobachten, wie Krankenschwestern in Praxen zur Pflege diabetischer Füße untergebracht sind.

Angesichts dieser Evidenz, die alarmierende Zahlen in Bezug auf diabetischen Fuß beinhaltet, basiert die Arbeit auf der folgenden Problemfrage: Welche Pflege entwickeln Pflegekräfte bei Patienten mit diabetischen Füßen?

Die fragliche Studie zielt darauf ab, die Pflege von diabetischem Fuß im Zusammenhang mit der Praxis von Krankenschwestern zu belegen. 


\section{METHODIK}

Für diese Studie wurde eine integrative Literaturrecherche durchgeführt. Laut Mendes; Silveira und Galvão (2019), die integrative Review-Methode wird nach klar definierten Schritten entwickelt; diese sind: Identifizierung des Themas und Auswahl der Leitfrage; Festlegung von Ein- und Ausschlusskriterien; Identifizierung vorausgewählter und ausgewählter Studien; Kategorisierung ausgewählter Studien; Analyse und Interpretation der Ergebnisse und Präsentation der Review-Synthese.

Der erste Schritt der integrativen Überprüfung ist die Nordfrage, die zu den aufgeführten Themen und Zielen führt. Daher stellt sich in diesem Artikel die Frage: Welche Pflege entwickeln Pflegekräfte bei Patienten mit diabetischen Füßen?

Um die Einschlusskriterien festzulegen, wurden verwendet: Artikel, die veröffentlicht wurden und zum Lesen zur Verfügung standen, da das Thema die Krankenschwester in ihren Zuschreibungen widerspiegelt, wurde die Datenbank von BDENF, LILACS und SCIELO ausgewählt, die zeitliche Abgrenzung erfolgte in den Jahren 2016 bis 2020, dh in den letzten fünf Jahren, und die portugiesische Sprache wurde für diese Aufnahme ausgewählt.

Für die Ausschlusskriterien wurden gestrichen: Artikel mit Doppelzüngigkeit, unvollständige Artikel, Annais, Meinungstexte, Editorials und Leserbriefe, Übersichtsartikel, Abschlussarbeiten, Dissertationen, Volltexte, die sich aber nicht auf das Thema beziehen.

Der Datenerhebungszeitraum entsprach dem Monat September 2021. Die identifizierten nationalen Deskriptoren waren: "Pflege", "diabetischer Fuß", "Pflege", "Diabetes mellitus". Der boolesche Operator andwurde verwendet, um die Deskriptoren in der Suchstrategie in der Datenbankplattform zuzuordnen.

Für die Auswahl ausgewählter und vorausgewählter Studien wurde eine gründliche Lektüre der Titel und Zusammenfassungen der gefundenen Artikel und dann eine vollständigere Lektüre im gesamten Artikel durchgeführt, wobei in seinen 
Ergebnissen nach der Identifizierung gesucht wurde, um das in diesem Artikel vorgeschlagene Ziel zu erreichen.

Es wurden zwei Suchen durchgeführt, die erste verwendete die folgende Suchstrategie: Pflege and diabetischer Fuß and Diabetes mellitus, mit dieser Strategie wurden 186 Artikel gefunden. Nach der Verwendung der Filter: vollständiger und verfügbarer Text, Datenbank, portugiesische Sprache und zeitliche Abgrenzung der letzten fünf Jahre, blieben 24 Artikel für die Förderfähigkeit übrig. Die zweite Suche wurde mit der folgenden Strategie durchgeführt: Krankenschwester and diabetischer Fuß fand 95 Artikel. Nach der Verwendung der Filter blieben 11 Artikel für die Berechtigung übrig.

Die Studien wurden aus einem synoptischen Bild kategorisiert und die Analyse und Präsentation der Ergebnisse wurde nach der Kategorisierung durchgeführt, die die Bedeutungskerne der Studien identifizierte und definierte, und dass aus der thematischen Analyse von Minayo (2012) eine Diskussion unter den Autoren gefunden wurde.

Für die Synthese des Wissens wurde eine Analyse der Limitationen, der Repräsentativität der Studie für die Gemeinschaft im Allgemeinen (Relevanz) und zukünftiger Studien durchgeführt, die im Verlauf dieses Artikels identifiziert wurden. Alle in dieser Studie verwendeten Produkte werden streng nach den Normen der Brasilianischen Vereinigung für Technische Normen (ABNT) referenziert.

\section{ERGEBNISSE UND DISKUSSIONEN}

Die Artikel, die für die Berechtigung mit den beiden Strategien übrig blieben, beliefen sich auf insgesamt 35 Artikel. Zunächst wurden Übersichtsartikel, Kurs Abschlussarbeiten, Leserbriefe, Erfahrungsberichte, Fallstudien, doppelte Artikel entfernt, die in Titel, Abstract und vollständiger Lektüre die Pflegekraft und ihre Assistenz nicht thematisiert haben, Hinterlassen von 07 Artikeln für diese Rezension. 
Zur besseren Veranschaulichung der gefundenen Ergebnisse enthält die folgende Tabelle die Charakterisierung der ausgewählten Studien. Die Daten beziehen sich auf: Artikeltitel, Erscheinungsjahr, Datenbank, Zeitschrift, Ort, Zielsetzung und Ergebnisse.

Tabelle 1. In den Datenbanken BDENF, LILACS und SCIELO gefundene Artikel zur Pflege von Patienten mit diabetischem Fuß.

\begin{tabular}{|c|c|c|c|c|c|}
\hline $\begin{array}{l}\text { Titel/ } \\
\text { Erscheinun } \\
\text { gsjahr }\end{array}$ & $\begin{array}{l}\text { Daten } \\
\text { bank }\end{array}$ & $\begin{array}{l}\text { Periodi } \\
\text { sche }\end{array}$ & Ziel & Lokal & Befund \\
\hline $\begin{array}{l}\text { Conhecim } \\
\text { ento do } \\
\text { enfermeiro } \\
\text { acerca dos } \\
\text { cuidados } \\
\text { com o pé } \\
\text { diabético } \\
2019\end{array}$ & $\begin{array}{l}\text { BDEN } \\
F\end{array}$ & $\begin{array}{l}\text { Rev. } \\
\text { enferm } \\
\text {. UFPE } \\
\text { on line }\end{array}$ & $\begin{array}{l}\text { Verständnis des } \\
\text { Wissens der } \\
\text { Krankenschwest } \\
\text { er über die } \\
\text { diabetische } \\
\text { Fußpflege in der } \\
\text { Primärversorgun } \\
\text { g }\end{array}$ & $\begin{array}{l}\text { Strategie für } \\
\text { Familiengesun } \\
\text { dheit/ESF }\end{array}$ & $\begin{array}{l}\text { Führen Sie } \\
\text { regelmäßig } \\
\text { eine } \\
\text { körperliche } \\
\text { Untersuchung } \\
\text { der Füße bei } \\
\text { Diabetikern } \\
\text { durch; } \\
\text { Einsatz von } \\
\text { Instrumenten } \\
\text { zur } \\
\text { Beurteilung } \\
\text { des } \\
\text { diabetischen } \\
\text { Fußes } \\
\text { Selbstfürsorge } \\
\text { zu füßen }\end{array}$ \\
\hline $\begin{array}{l}\text { Riscos } \\
\text { associados } \\
\text { à }\end{array}$ & $\begin{array}{l}\text { LILAC } \\
\text { S/ } \\
\text { BDEN }\end{array}$ & $\begin{array}{l}\text { Rev. } \\
\text { gaúch. } \\
\text { enferm }\end{array}$ & $\begin{array}{l}\text { Identifizieren Sie } \\
\text { bei Patienten mit } \\
\text { Typ-2-Diabetes, }\end{array}$ & $\begin{array}{l}\text { Aufklärungspro } \\
\text { gramm zur } \\
\text { Prävention und }\end{array}$ & $\begin{array}{l}\text { Gesundheitse } \\
\text { rziehung über } \\
\text { Fußhygiene, }\end{array}$ \\
\hline
\end{tabular}


REVISTA CIENTIFICA MULTIDISCIPLINAR NÚCLEO DO CONHECIMENTO ISSN: 2448-0959

https://www.nucleodoconhecimento.com.br

\begin{tabular}{|c|c|c|c|c|c|}
\hline $\begin{array}{l}\text { mortalidad } \\
\text { e em } \\
\text { pacientes } \\
\text { atendidos } \\
\text { em um } \\
\text { programa } \\
\text { de } \\
\text { prevenção } \\
\text { do pé } \\
\text { diabético } \\
2018\end{array}$ & $F$ & & $\begin{array}{l}\text { welche } \\
\text { Fußveränderung } \\
\text { en mit } \\
\text { demografischen, } \\
\text { klinischen, } \\
\text { biochemischen } \\
\text { und } \\
\text { behandlungsbedi } \\
\text { ngten Merkmalen } \\
\text { einhergehen und } \\
\text { das } \\
\text { Mortalitätsrisiko } \\
\text { erhöhen würden }\end{array}$ & $\begin{array}{l}\text { Behandlung } \\
\text { des } \\
\text { diabetischen } \\
\text { Fußes in einem } \\
\text { Allgemein- und } \\
\text { Universitätsklini } \\
\text { kum von hoher } \\
\text { Komplexität }\end{array}$ & $\begin{array}{l}\text { die Wahl } \\
\text { geeigneter } \\
\text { Schuhe, das } \\
\text { beste } \\
\text { Verhalten in } \\
\text { Notsituationen } \\
\text { Systemische } \\
\text { Versorgung } \\
\text { wie } \\
\text { Nichtrauchen, } \\
\text { Aufrechterhalt } \\
\text { ung einer } \\
\text { guten } \\
\text { glykämischen } \\
\text { Kontrolle, } \\
\text { Kontrolle der } \\
\text { Blutfettwerten } \\
\text { und der } \\
\text { korrekten } \\
\text { Verwendung } \\
\text { von } \\
\text { Medikamente } \\
\mathrm{n}\end{array}$ \\
\hline $\begin{array}{l}\text { Condutas } \\
\text { dos } \\
\text { enfermeiro } \\
\text { s da } \\
\text { atenção } \\
\text { primária no }\end{array}$ & $\begin{array}{l}\text { BDEN } \\
F\end{array}$ & $\begin{array}{l}\text { Rev. } \\
\text { enferm } \\
\text {. UFPE } \\
\text { on line }\end{array}$ & $\begin{array}{l}\text { Die Handlungen } \\
\text { von } \\
\text { Krankenschwest } \\
\text { ern in der } \\
\text { Grundversorgun } \\
\mathrm{g} \text { bei der Pflege }\end{array}$ & $\begin{array}{l}\text { Strategie für } \\
\text { Familiengesun } \\
\text { dheit }\end{array}$ & $\begin{array}{l}\text { Häufigkeit und } \\
\text { Terminierung } \\
\text { von } \\
\text { Beratungen, } \\
\text { Servicedynam } \\
\text { ik, Kriterien für }\end{array}$ \\
\hline
\end{tabular}

RC: 105166

Verfügbar in: https://www.nucleodoconhecimento.com.br/gesundheit/diabetischenfuss 


\begin{tabular}{|c|c|c|c|c|c|}
\hline $\begin{array}{l}\text { cuidado a } \\
\text { pessoas } \\
\text { com pé } \\
\text { diabético } \\
2017\end{array}$ & & & $\begin{array}{l}\text { von Menschen } \\
\text { mit Diabetes } \\
\text { mellitus (DM) im } \\
\text { Zusammenhang } \\
\text { mit diabetischem } \\
\text { Fuß zu kennen }\end{array}$ & & $\begin{array}{l}\text { die } \\
\text { Fußuntersuch } \\
\text { ung, } \\
\text { Selbstversorg } \\
\text { ung }\end{array}$ \\
\hline $\begin{array}{l}\text { Ações do } \\
\text { enfermeiro } \\
\text { na } \\
\text { prevenção } \\
\text { do pé } \\
\text { diabético: } \\
\text { o olhar da } \\
\text { pessoa } \\
\text { com } \\
\text { diabetes } \\
\text { mellitus } \\
2017\end{array}$ & $\begin{array}{l}\text { LILAC } \\
\text { S/ } \\
\text { BDEN } \\
\text { F }\end{array}$ & $\begin{array}{l}\text { Rev. } \\
\text { Pesqui. } \\
\text { (Univ. } \\
\text { Fed. } \\
\text { Estado } \\
\text { Rio J., } \\
\text { Online) }\end{array}$ & $\begin{array}{l}\text { Untersuchen Sie } \\
\text { die von } \\
\text { Krankenschwest } \\
\text { ern } \\
\text { durchgeführten } \\
\text { Maßnahmen zur } \\
\text { Prävention von } \\
\text { diabetischen Fuß } \\
\text { aus der } \\
\text { Perspektive der } \\
\text { Person mit DM. }\end{array}$ & $\begin{array}{l}\text { Hochschule } \\
\text { Erweiterungspr } \\
\text { ojekt }\end{array}$ & $\begin{array}{l}\text { Spezifische } \\
\text { Aktionen, die } \\
\text { die } \\
\text { Fußuntersuch } \\
\text { ung, den } \\
\text { kommunikativ } \\
\text { en Dialog, die } \\
\text { Gesundheitse } \\
\text { rziehung } \\
\text { umfassen. }\end{array}$ \\
\hline $\begin{array}{l}\text { Alterações } \\
\text { nos pés do } \\
\text { idoso } \\
\text { hospitaliza } \\
\text { do: um } \\
\text { olhar } \\
\text { cuidadoso } \\
\text { da } \\
\text { enfermage } \\
\text { m } \\
2017\end{array}$ & $\begin{array}{l}\text { LILAC } \\
\text { S/ } \\
\text { BDEN } \\
\text { F }\end{array}$ & $\begin{array}{l}\text { Esc. } \\
\text { Anna } \\
\text { Nery } \\
\text { Rev. } \\
\text { Enferm }\end{array}$ & $\begin{array}{l}\text { Charakterisierun } \\
\mathrm{g} \text { des } \\
\text { pädagogischen } \\
\text { Profils älterer } \\
\text { Menschen, die } \\
\text { auf den } \\
\text { Stationen eines } \\
\text { Universitätskrank } \\
\text { enhauses } \\
\text { hospitalisiert } \\
\text { sind; } \\
\text { Identifizieren Sie }\end{array}$ & Kra & $\begin{array}{l}\text { Stärkung der } \\
\text { Bindungen zu } \\
\text { diesen } \\
\text { Diabetikern, } \\
\text { so dass sie } \\
\text { zur Post } \\
\text { kommen, um } \\
\text { häufig } \\
\text { Fußuntersuch } \\
\text { ungen } \\
\text { durchzuführen } \\
\text { Ausbildung }\end{array}$ \\
\hline
\end{tabular}




\begin{tabular}{|c|c|c|c|c|c|}
\hline & & & $\begin{array}{l}\text { die Fußpflege } \\
\text { Anforderungen } \\
\text { von älteren } \\
\text { Patienten im } \\
\text { Krankenhaus; } \\
\text { und die } \\
\text { Handlungsmöglic } \\
\text { hkeiten von } \\
\text { Krankenschwest } \\
\text { ern mit diesen } \\
\text { älteren } \\
\text { Menschen zu } \\
\text { analysieren. }\end{array}$ & & $\begin{array}{l}\text { und } \\
\text { Qualifizierung } \\
\text { zur } \\
\text { Unterstützung } \\
\text { dieser } \\
\text { Probleme, } \\
\text { vermeidung } \\
\text { größerer } \\
\text { Komplikatione } \\
\text { n und } \\
\text { Reduzierung } \\
\text { zukünftiger } \\
\text { Pflegeanforde } \\
\text { rungen }\end{array}$ \\
\hline $\begin{array}{l}\text { Atuação } \\
\text { dos } \\
\text { enfermeiro } \\
\text { s da } \\
\text { estratégia } \\
\text { saúde da } \\
\text { família na } \\
\text { prevenção } \\
\text { do pé } \\
\text { diabético } \\
2016\end{array}$ & $\begin{array}{l}\text { LILAC } \\
\text { S/ } \\
\text { BDEN } \\
\text { F }\end{array}$ & $\begin{array}{l}\text { Rev. } \\
\text { Pesqui. } \\
\text { (Univ. } \\
\text { Fed. } \\
\text { Estado } \\
\text { Rio J., } \\
\text { Online) } \\
\text {; }\end{array}$ & 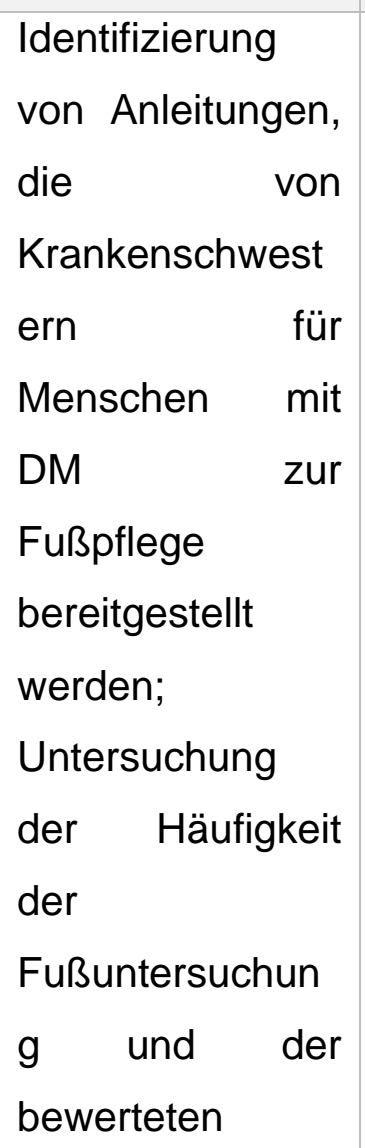 & $\begin{array}{l}\text { Strategie für } \\
\text { Familiengesun } \\
\text { dheit }\end{array}$ & $\begin{array}{l}\text { Gesundheitse } \\
\text { rziehung für } \\
\text { Diabetiker, } \\
\text { Untersuchung } \\
\text { der Füße von } \\
\text { Menschen mit } \\
\text { DM, Bildung } \\
\text { von } \\
\text { Koexistenzgru } \\
\text { ppen, } \\
\text { Verbände in } \\
\text { Techniken, die } \\
\text { der Art der } \\
\text { Fußverletzung } \\
\text { entsprechen. }\end{array}$ \\
\hline
\end{tabular}

RC: 105166

Verfügbar in: https://www.nucleodoconhecimento.com.br/gesundheit/diabetischenfuss 


\begin{tabular}{|c|c|c|c|c|c|}
\hline & & & $\begin{array}{l}\text { Aspekte; um zu } \\
\text { überprüfen, } \\
\text { welche } \\
\text { Gesundheitserzi } \\
\text { ehung Aktivitäten } \\
\text { Krankenschwest } \\
\text { ern für } \\
\text { Menschen mit } \\
\text { DM durchführen. }\end{array}$ & & \\
\hline $\begin{array}{l}\text { A } \\
\text { perspectiv } \\
\text { a da } \\
\text { vulnerabili } \\
\text { dade na } \\
\text { avaliação } \\
\text { do pé } \\
\text { diabético } \\
\text { sob a ótica } \\
\text { de } \\
\text { enfermeiro } \\
\text { s } \\
2016\end{array}$ & $\begin{array}{l}\text { BDEN } \\
\mathrm{F}\end{array}$ & $\begin{array}{l}\text { Cogitar } \\
\text { e } \\
\text { enferm }\end{array}$ & $\begin{array}{l}\text { Schreiben Sie } \\
\text { die sozialen } \\
\text { Determinanten } \\
\text { Bedingungen in } \\
\text { der Gesundheit, } \\
\text { die von } \\
\text { Krankenschwest } \\
\text { ern als relevant } \\
\text { für die } \\
\text { Feststellung von } \\
\text { Schwachstellen } \\
\text { der Person mit } \\
\text { Diabetes mellitus } \\
\text { identifiziert } \\
\text { wurden. }\end{array}$ & $\begin{array}{l}\text { Zufällig } \\
\text { ausgewählte } \\
\text { Krankenschwe } \\
\text { stern in Curitiba }\end{array}$ & $\begin{array}{l}\text { Stimulieren } \\
\text { Sie den } \\
\text { Diabetiker, zur } \\
\text { Ernährungsbe } \\
\text { ratung zu } \\
\text { gehen und die } \\
\text { Diät zu } \\
\text { befolgen. }\end{array}$ \\
\hline
\end{tabular}

Quelle: Autor.

Die Studie erwähnt in ihren Zielen drei Arten der Pflege, die von Krankenschwestern für Patienten mit diabetischen Füßen durchgeführt werden: Die erste, die am meisten entstanden ist, basiert auf Maßnahmen, die auf die Gesundheitserziehung als Grundprinzip der Krankheitsprävention und ihrer Komplikationen abzielen, die zweite

RC: 105166

Verfügbar in: https://www.nucleodoconhecimento.com.br/gesundheit/diabetischen$\underline{\text { fuss }}$ 
Pflege basiert auf Fußbewertungstechniken bei Diabetikern als eine Möglichkeit, komplikationen zu entstehen, und die dritte Pflege konzentriert sich auf Krankenschwestern als Stimulator und Motivator Diabetiker zu ihrer Selbstversorgung mit den Füßen. Diese drei Vorsichtsmaßnahmen werden in ihren Themen entsprechend den ausgewählten Studien diskutiert.

\subsection{GESUNDHEITSERZIEHUNG ALS PRÄVENTIONSFORM}

Daher kann der Patient aus der Gesundheitserziehung die Gründe verstehen, warum der Wundheilungsprozess langsam ist, die Gründe für die Hauptkomplikationen und insbesondere das Wissen über die Risikoprävention. Krankenschwestern sollten in der Lage sein, die Bedürfnisse des Patienten zu erkennen und so zu genauen und sicheren Pflegediagnosen im Zusammenhang mit der Wunde in ihrem Stadium beizutragen.

Scain; Franzen; Hirakata (2018) berichtet, dass Krankenschwestern bemühungen, Gesundheitserziehung mit Patienten durchzuführen, hartnäckig und beharrlich geworden sind, um den Bildungsprozess bei Patienten mit diabetischen Füßen fortzusetzen, Kreativität bei der Entwicklung von Bildungstechnologien wie Ordnern, Videos zu nutzen und zur Verfügung zu stehen, um Fragen zu einem abnormalen Zustand mit dem Patienten von Social-Media-Technologien wie Whatsapp / Telefon zu stellen.

Camillo et al. (2016) erwähnt, dass das Konzept der Gesundheitserziehung über das Einbringen von Daten oder sogar die Beseitigung von Zweifeln hinausgeht, betrachten die Autoren die Gesundheitserziehung als einen systematischen, kontinuierlichen und dauerhaften Prozess, der auf die Bildung und Entwicklung eines kritischen Bewusstseins des Bürgers abzielt, durch die Suche nach kollektiven Lösungen für die erlebten Probleme und ihre "echte Beteiligung" an der Ausübung der sozialen Kontrolle. 
Vargas et al. (2017) bringt Gesundheitserziehung als einen der Hauptzwecke der Zuschreibung von Krankenschwestern an die Pflege, die Autoren bekräftigen, dass die Gesundheitserziehung dem Patienten eine proaktive Haltung begünstigt und zeigt, dass der Patient Sicherheit bei der Pflege des diabetischen Fußes haben sollte.

Autonomie und Sicherheit in der Pflege wurden auch in der Studie von Pereira et al. (2017) weisen die Autoren darauf hin, dass die Gesundheitserziehung zum wichtigsten Mechanismus zur Vorbeugung von Krankheiten und ihren Komplikationen wird, in Bezug auf den diabetischen Fuß fördert die Gesundheitserziehung die Sicherheit, das Vertrauen und die Autonomie der Patienten.

Der Nachweis, dass Gesundheitserziehung wirksam ist, wurde in einer deskriptiven Studie des quantitativen Ansatzes identifiziert, in der gezeigt wurde, dass gesundheitserziehung den Patienten ein theoretisches Wissen über die Krankheit vermittelt, das die Kultur und den Glauben der Patienten respektiert, und dass die Förderung von Bildungsprozessen nicht nur gemeinsam, sondern auf individualisierte Weise stattfindet. die Zweifel zu beseitigen, die den Patienten während der pflegerischen Konsultation oder sogar in einem informellen Dialog zwischen dem Patienten und dem Fachmann durchdringen (DE OLIVEIRA et al., 2016).

Aber Gesundheitserziehung wird nicht nur als ein Prozess der Pflege für Patienten identifiziert, Krankenschwestern sollten auch auf Ausbildung und Qualifikationen achten, die auch auf technischere Weise als Gesundheitserziehung betrachtet werden können. Silva; Santo und Chibante (2017) stellt fest, dass Krankenschwestern immer auf neue Wege der Pflege diabetischer Füße auf dem Laufenden gehalten werden sollten, da Behandlungsprozesse zunehmend in der wissenschaftlichen Literatur untersucht werden und Innovationen für die Heilung oder sogar Wund Inszenierung bringen. 
Daher sind Gesundheitserziehung, Fort- und Weiterbildung Formen der Pflege, mit denen Pflegekräfte das Krankheitsbild von Patienten mit diabetischen Füßen verbessern.

\subsection{TECHNIKEN ZUR BEWERTUNG VON FÜSSEN BEI DIABETISCHEN FÜSSEN}

Die in der Ausbildung zum Pflegepersonal und im Rahmen der Fort- und Weiterbildung erworbenen technisch-wissenschaftlichen Kenntnisse sind relevante Faktoren für die Behandlung des diabetischen Fußes, daher ist es Aufgabe des Pflegepersonals, sich Kenntnisse zur Durchführung sicherer Techniken zur Beurteilung von Patienten mit Diabetes anzueignen als Methode zur Vorbeugung und für Patienten mit diabetischem $F u ß$ als vorbeugende Maßnahme für Komplikationen.

Arruda et al. (2019) besagt, dass die körperliche Untersuchung von diabetischen Füßen Verfahren sind, die von Krankenschwestern durchgeführt werden, um mögliche Komplikationen wie die Entwicklung von Geschwüren und Amputationen der unteren Gliedmaßen zu vermeiden. Daher induziert die Unkenntnis dieser Technik die höheren Komplikationsraten. Die genannten Autoren weisen zu einem anderen Zeitpunkt darauf hin, dass neben der Technik, die aufgrund mangelnder Kenntnisse der Krankenschwester nicht angewendet wird, auch die Arbeitsbedingungen die Nichtleistung der Bewertungstechnik beeinflussen, diese Bedingungen werden von den Autoren als unzureichende Infrastruktur und Forderung nach übermäßiger Pflege bezeichnet.

Vargas et al. (2017) bringt in seiner Studie einen ähnlichen Kontext wie die zuvor erwähnte Studie und fügt hinzu, dass Krankenschwestern mehr Training in Bezug auf Bewertungstechniken im Zusammenhang mit diabetischem Fuß benötigen, Krankenschwestern berichten auch, dass das Management keine Materialien anbietet, um diese Techniken durchzuführen und über über überzogene Anforderungen zu sprechen.

RC: 105166

Verfügbar in: https://www.nucleodoconhecimento.com.br/gesundheit/diabetischenfuss 
Pereira et al. (2017) ist der Ansicht, dass spezifische Maßnahmen ergriffen werden sollten, um das Versorgungssystem für Patienten mit diabetischem Fuß neu zu gestalten, wobei diese Maßnahmen über die Fußuntersuchung hinausgehen und eine wirksame Kommunikation und Gesundheitserziehung bieten sollten.

Leal et al. (2016) ist der Ansicht, dass Krankenschwestern aufgrund mehrerer Faktoren unwissend über das Thema sein können, und einer von innen wird von den Autoren als der hohe Bedarf an Pflege hervorgehoben. Diese Forderung impliziert eine körperliche und geistige Erschöpfung des Fachmanns, die einen Mangel an Zeit für die berufliche Qualifikation bietet. In einem anderen Sinne erwähnen die Autoren, dass die übermäßige Nachfrage nach einem bestimmten Problem dem Fachmann raum für eine permanente Gesundheitserziehung bietet, in der diese Art von Ausbildung Transformationen in der beruflichen Praxis vorschlägt, die auf bestehenden Problemen im Prozess ihrer Arbeit basieren und lernen, die Praxis in ihrer Realität widerzuspiegeln.

Krankenpflege trägt die Funktion mit sich, Erkrankungen der Haut und ihrer angrenzenden Krankheiten zu behandeln. Dies liegt daran, dass Krankenschwestern in ihren Zuschreibungen die Pflege des Patienten und die Notwendigkeit haben, diese Pflege im Zusammenhang mit haut bedingten Krankheiten zu erweitern (DA SILVA BRANDAO et al., 2016; SA et al., 2016).

\subsection{DIE VERMITTLUNG KRANKENSCHWESTER ZUR SELBSTVERSORGUNG FÜR PATIENTEN MIT DIABETISCHER FUß}

Von Selbstfürsorge zu sprechen bezieht sich auf jede Handlung, die sich aus dem täglichen Handeln des Menschen ergibt. Was die Selbstfürsorge betrifft, so haben Arruda et al. (2019) berichten, dass Patienten mit diabetischem Fuß dazu neigen, eine große wirtschaftliche, soziale, kulturelle, emotionale und biologische Wirkung zu erzielen, die eine größere Abhängigkeit von anderen begünstigt, um bei den Aktivitäten des täglichen Lebens zu helfen, und auswirkungen auf ihre Selbstversorgung und Lebensqualität. 
Scain;. Franzen und Hirakata (2018) berichten, dass der Patient sich der Durchführung seiner eigenen Aktivitäten bewusst sein sollte und dass die Selbstversorgung kontinuierlich sein sollte, eine der von den Autoren zitierten Möglichkeiten, die Selbstpflege durchzuführen, war: Hygiene der Füße; Wahl des geeigneten Schuhwerks; Vermeiden Sie es, barfuß und andere zu gehen.

An Vargas et al. (2017) bringt als Selbstversorgung die Aufrechterhaltung der glykämischen Kontrolle, die proaktive Teilnahme von Patienten an der Durchführung von Fußuntersuchungen, Therapie, absolute Beschränkung der Verwendung von Tabak und Getränken, Ernährung, körperliche Bewegung, körperliche Aktivität und andere Formen, die die zuvor erwähnte Studie in Betracht ziehen.

Eine Möglichkeit, den Patienten dazu zu bringen, sich um die Selbstfürsorge zu bemühen, wird von den Autoren Silva erwähnt; Santo und Chibante (2017) als Stärkung der Bindungen zwischen Patient und medizinischem Fachpersonal. Diese Stärkung fördert die Sicherheit und Glaubwürdigkeit des Patienten, dass durch kontinuierliche und systematische Selbstpflege an den Füßen das Risiko von Komplikationen geringer ist.

Die Autonomie und Sicherheit des Patienten mit diabetischem Fuß minimieren Schäden und das Risiko von Komplikationen. Ein Patient, der an der Behandlung seiner Krankheit beteiligt ist, neigt dazu, sein Wissen zu erweitern, um die Abhängigkeit von anderen Menschen zu vermeiden, und artikuliert die Selbstfürsorge in seinen Aktivitäten als eine Form der Motivation und Demonstration der Fähigkeit angesichts von Widrigkeiten, die mit der Krankheit einhergehen (DE OLIVEIRA et al., 2016).

An Fatima Bento et al. (2016) leiden Patienten mit diabetischen Füßen bereits unter Stigmata aufgrund ihres Zustands, was es für sie schwierig macht, eine angemessene Versorgung zu haben, da die Bedingungen, die eine Distanzierung von der Gesundheitseinheit, Schwierigkeiten bei der Mobilisierung, wenig Wissen 
über die Krankheit, eine unzureichende Versorgung und die Entwicklung von Komplikationen beinhalten.

Nascimento et al. (2016) erwähnen, dass, wenn qualifizierte und humanisierte Pflege durch effektives Verhalten mit einfachem Zugang zu Gesundheitsdiensten bereitgestellt wird, in die alle Maßnahmen auf allen Ebenen der Versorgung integriert werden können, Selbstversorgung ohne Risiko von Komplikationen.

\section{FAZIT}

Diese Studie reflektierte die Pflege von Krankenschwestern und Fachleuten in der Leistung der Pflege und Pflege von Patienten mit diabetischem Fuß. Die relevanten Versorgungsstudien identifizierten, was zur Realisierung von drei Themen für die Diskussion der gefundenen Ergebnisse führte.

Das Ziel der Studie zielte darauf ab, die Pflege von Krankenschwestern für Patienten mit diabetischen Füßen hervorzuheben, es wird betont, dass die Pflege von Krankenschwestern und nicht andere Kategorien der Krankenpflege betont wurde. Die Studien zeigten Gesundheitserziehung als erste Versorgung, was nicht widerlegt werden kann, da die Literatur betont, dass Gesundheitserziehung für die Krankheitsprävention von größter Bedeutung ist.

Eine andere Art der Versorgung spiegelt sich im eigenen Wissen des Fachmanns über den diabetischen Fuß wider, der innerhalb des Gesundheitssystems von äußerster Notwendigkeit und großer Sorge ist, da die Artikel zu Überlegungen führten, dass Pflegekräfte qualifizierter sein sollten, um die Versorgung des Patienten mit mehr Exzellenz zu leiten .

Eine wichtige Sorgfalt, auf die auch durch die Studien hingewiesen wird und die direkt mit der ständigen oder kontinuierlichen Weiterbildung von Fachleuten geleitet wird, sind die Techniken der körperlichen Untersuchung, bei dieser Art der Pflege sollte die Krankenschwester in der Lage sein, die Untersuchung von diabetischen 
Füßen weise und sicher durchzuführen, neben der Ermutigung der Patienten, häufiger zur Untersuchung zu gehen.

In Bezug auf die Pflege im Zusammenhang mit der eigenen Selbstversorgung des Patienten spiegelte sich wider, dass Fußhygiene, glykämische Kontrolle, kontinuierliche Nachsorge mit Fußuntersuchung, die Verwendung geeigneter Schuhe und andere eine größere Prävention gegen Komplikationen unter anderem die Amputation von Gliedmaßen verstärken.

Es wird daher der Schluss gezogen, dass die Studie mehrere Formen der Pflege durch Krankenschwestern identifiziert hat und dass alle diese Formen für die Kontinuität der Behandlung und Prävention und Komplikationen wichtig sind, aber es liegt an der Krankenschwester, ein Fachmann zu sein, der die Qualität seiner Pflege auf der Grundlage theoretischer Konzepte schätzt, die zu häufigen Schulungen führen.

\section{VERWEISE}

AMERICAN DIABETES ASSOCIATION. Standards of medical care in diabetes. Diabetes Care, v. 40, n. suppl 1, p. s1-s128, 2017.

ARRUDA, Cecilia et al. Tecnologia educativa para cuidados e prevenção do pé diabético/Educational technology for care and prevention of diabetic foot ulcers. Ciência, Cuidado e Saúde, v. 20, 2021.

ARRUDA, Luana Savana Nascimento de Sousa et al. Conhecimento do enfermeiro acerca dos cuidados com o pé diabético. Rev. enferm. UFPE on line, p. [1-8], 2019.

ASCHNER M, Pablo et al. Clinical practice guideline for the prevention, early detection, diagnosis, management and follow up of type 2 diabetes mellitus in adults. Colomb. Med., Cali, v. 47, n. 2, p. 109-130, junho. 2016. 
CAMILLO, Bibiana Schultz et al. Ações de educação em saúde na atenção primária a gestantes e puérperas: revisão integrativa. Revista de enfermagem UFPE on line-ISSN: 1981-8963, v. 10, n. 6, p. 4894-4901, 2016.

CUBAS, Marcia Regina et al. Pé diabético: orientações e conhecimento sobre cuidados preventivos. Fisioterapia em movimento, v. 26, n. 3, 2017.

DE FÁTIMA BENTO, Leandra et al. A perspectiva da vulnerabilidade na avaliação do pé diabético sob a ótica de enfermeiros. Cogitare Enfermagem, v. 21, n. 1, 2016.

DE OLIVEIRA, Patrícia Simplício et al. Atuação dos enfermeiros da Estratégia Saúde da Família na prevenção do pé diabético Practice nurse family health strategy in the prevention of diabetic foot. Revista de Pesquisa Cuidado é Fundamental Online, v. 8 , n. 3, p. 4841-4849, 2016.

DE SOUZA MENDONÇA, Sarah; MORAIS, Juliana de Sant'Anna; DE MOURA, Maria Catarina Gomes Gadelha. Proposta de um protocolo de avaliação fisioterapêutica para os pés de diabéticos. Fisioterapia em Movimento, v. 24, n. 2, 2017.

FELIX, Lidiany Galdino et al. Conhecimento de enfermeiros da atenção primária antes e após intervenção educativa sobre pé diabético. Revista Gaúcha de Enfermagem, v. 42, 2021.

LEAL, Maria do Carmo et al. Atenção ao pré-natal e parto em mulheres usuárias do sistema público de saúde residentes na Amazônia Legal e no Nordeste, Brasil 2010. Rev. Bras. Saude Mater. Infant. Recife, v. 15, n. 1, p. 91-104, Mar. Available from \&lt; http://www.scielo.br/scielo.php?script=sci_arttext\&amp;pid=S151938292015000100091\&amp; Ing=en\&amp;nrm=iso\&gt;.

MENDES, Karina Dal Sasso; SILVEIRA, Renata Cristina de Campos Pereira; GALVÃO, Cristina Maria. Uso de gerenciador de referências bibliográficas na 
seleção dos estudos primários em revisão integrativa. Texto \& ContextoEnfermagem, v. 28, 2019.

MINAYO, Maria Cecília de Souza. Análise qualitativa: teoria, passos e fidedignidade. Ciência \& saúde coletiva, v. 17, p. 621-626, 2012.

MUZY, Jéssica et al. Prevalência de diabetes mellitus e suas complicações e caracterização das lacunas na atenção à saúde a partir da triangulação de pesquisas. Cadernos de Saúde Pública, v. 37, 2021.

NASCIMENTO, Vagner Ferreira do et al. Percepção de puérperas sobre as primeiras consultas de pré-natal no interior de Mato Grosso. Rev. enferm. UFPI, v.5, n. 1, p. 46-51, 2016.

ORGANIZAÇÃO MUNDIAL DE SAÚDE. Definition, diagnosis and classification of diabetes Mellitus and its complications. Geneva: OMS, 1999. Disponível em: https://apps.who.int/iris/bitstream/handle/10665/66040/WHO_NCD_NCS_99.2.pdf?s equence $=1$ \&isAllowed $=\mathrm{y}$

PEREIRA, Laiane de Fátima et al. Ações do enfermeiro na prevenção do pé diabético: o olhar da pessoa com diabetes mellitus. Rev. pesqui. cuid. fundam. (Online), p. 1008-1014, 2017.

SCAIN, Suzana Fiore; FRANZEN, Elenara; HIRAKATA, Vânia Naomi. Riscos associados à mortalidade em pacientes atendidos em um programa de prevenção do pé diabético. Revista Gaúcha de Enfermagem, v. 39, 2018.

SILVA, Caline Oliveira da. Análise da qualidade de vida em indivíduos com Diabetes Mellitus Tipo 1. 2017. 34 f. Trabalho de conclusão de curso (Bacharelado em Farmácia) -Universidade de Brasília, Brasília, 2017. Disponível em: https://bdm.unb.br/handle/10483/19301 
SILVA, Joziane Santos da; SANTO, Fátima Helena do Espírito; CHIBANTE, Carla Lube de Pinho. Alterações nos pés do idoso hospitalizado: um olhar cuidadoso da enfermagem. Escola Anna Nery, v. 21, 2017.

SOCIEDADE BRASILEIRA DE DIABETES. Tratamento e acompanhamento do diabetes Mellitus: Diretrizes da Sociedade Brasileira de Diabetes. 3.ed. Itapevi, SP: A. Araújo Silva Farmacêutica; 2017/2018. Disponível em: http://www.diabetes.org.br/ profissionais/images/2017/diretrizes/diretrizes-sbd-20172018.pdf.

VARGAS, Caroline Porcelis et al. Condutas dos enfermeiros da atenção primária no cuidado a pessoas com pé diabético. Rev. enferm. UFPE on line, p. 4535-4545, 2017.

Eingereicht: Oktober 2021.

Genehmigt: Dezember 2021. 